\title{
Can Sentinel Lymph Node Biopsy Predict Various Levels of Echelon Nodes in Oral Cancers?
}

\author{
Ramya Rathod ${ }^{1}$ Jaimanti Bakshi ${ }^{1}$ Naresh Kumar Panda ${ }^{1}$ Roshan Verma ${ }^{1}$ Anish Bhattacharya ${ }^{2}$ \\ Amanjit Bal ${ }^{3}$
}

${ }^{1}$ Department of Otolaryngology, Head \& Neck Surgery, PostGraduate Institute of Medical Education and Research, Chandigarh, Chandigarh, India

2 Department of Nuclear Medicine, Post-Graduate Institute of Medical Education and Research, Chandigarh, Chandigarh, India

${ }^{3}$ Department of Pathology, Post-Graduate Institute of Medical

Education and Research, Chandigarh, Chandigarh, India
Address for correspondence Ramya Rathod, MS, Department of Otolaryngology, Head and Neck surgery, Post graduate institute of medical education and research, Sector-12, Chandigarh, India (e-mail: ramyar2290@gmail.com).

Int Arch Otorhinolaryngol 2020;24:e125-e131.

\begin{abstract}
Keywords

- sentinel node

- oral cancer

- neck dissection

- lymphoscintigraphy

- technetium

Introduction The efficacy of sentinel node biopsy in early stage oral cancer is well established. Its evolving role can be reinforced by further studies.

Objective Analyzing the predictability of the levels of echelon nodes for various oral cavity tumor subsites on sentinel node biopsy.

Methods A prospective study of 20 patients with stage $1 / 11$ oral squamous cell carcinoma who underwent sentinel node biopsy-guided neck dissection between January 2017 and 2018 at our institute. The procedure included radiotracer injection, imaging (lymphoscintigraphy, single photon emission computed tomography-computed tomography), and gamma probe application. Sentinel node detection on imaging and gamma probe were compared.

Results Out of 20 patients, $13(65 \%)$ had carcinoma of the tongue, $6(30 \%)$ had buccal mucosa carcinoma, and $1(5 \%)$ had retromolar trigone carcinoma. The mean age of the patients was 52.3 years. A total of $13(65 \%)$ patients were male, and $7(35 \%)$ were female. The sentinel node identification rates with imaging and gamma probe were of $70 \%$ and $100 \%$ respectively. In tongue and retromolar trigone primaries, the most common first-echelon nodes in both modalities were levels IIA and IB respectively. For buccal mucosa primaries, first-echelon nodes were detected only with the gamma probe, which was level IB. On imaging, second-echelon nodes were detected only for tongue primaries, and had equivalent incidence of levels II, III, and IV. On the gamma probe, level IIA, followed by III, and IV for the tongue, and level IIA were the most common second-echelon nodes for the buccal mucosa. Third-echelon nodes were detected only with the gamma probe for tongue carcinoma at level IV.

Conclusion The combined use of imaging and gamma probe provides the best results, with high identification rate and predictability of echelon levels.
\end{abstract}

received

June 6, 2019

accepted

June 20, 2019
DOI https://doi.org/

10.1055/s-0039-1695762.

ISSN $1809-9777$.
Copyright $(2020$ by Thieme Revinter

Publicações Ltda, Rio de Janeiro, Brazil
License terms

() (i) $\ominus$ (\$) 


\section{Introduction}

Cervical lymph node (LN) metastasis is the single most important prognostic factor in squamous cell carcinoma of the head and neck, which can decrease the 5-year survival rates from $80 \%$ to $<50 \%$. None of the currently available imaging methods reliably depicts small tumor deposits in non-enlarged LNs or differentiates reactively enlarged LNs from metastatic adenopathy. Therefore, necks with oral cancer in stages I and II are managed when the risk of micrometastasis is $>30 \%$, in which case either radiotherapy or elective neck dissection (END) are performed. ${ }^{1}$ There is a paradigm shift from extensive to selective neck dissection (SND) for oral cavity cancers. But the rate of skip metastases to level IV is $\sim 10 \%$ for tongue primaries. Thus, the concept of sentinel LN biopsy (SLNB) came to be. It mimics the physiological migration of cancer cells from the tumor to the LNs, with migration of a known detectable tracer. ${ }^{2}$

Technetium $99 \mathrm{~m}$ (Tc $99 \mathrm{~m}$ ) sulfur colloid is the most commonly used radiotracer agent, with a physical half-life of 6 hours. $^{3}$ Depending on the size of the particle, the radiotracer can move down the lymphatics from the first-echelon node to the second and third-echelon nodes. Smaller particles drain quickly from the sentinel node, and tend to accumulate in non-sentinel nodes, whereas larger particles drain slowly and are retained within the sentinel nodes. ${ }^{4}$

The gamma camera interacts with the gamma rays emitted from the radionuclide injection site, and gives total radiotracer uptake and its spatial distribution within the tissue. ${ }^{5}$ Single photon emission computed tomography-computed tomography (SPECT-CT) gives better definition to the images, with the location of the LNs relative to anatomical landmarks, and also reduces the obscuring of nodes by activity of an injection site that is near the tumor. ${ }^{6}$

In the present study, our objective was to determine the ability of this modality to predict first-, second-, and thirdechelon node levels for various tumor subsites of the oral cavity.

\section{Methods}

The study was conducted at the Department of Otolaryngology and Head \& Neck Surgery in collaboration with the Department of Nuclear Medicine and the Department of Pathology at our institute. This was a prospective study with a total of 20 diagnosed patients of early-stage oral cancers. Prior approval was obtained from the ethics committee of the institution, with reference no. NK/4238/MS/ 2224-25. The treatment plan was discussed in detail with the patient and their attendants, and informed and written consent was sought before proceeding with the study. The minimum follow-up period planned was of 6 months, and the maximum follow-up period was variable.

All diagnosed cases of stages I and II oral cavity cancers were included, and patients with $\mathrm{T} 3$ or $\mathrm{T} 4$ diseases, irrespective of nodal status, with node-positive neck, with malignancy of multiple subsites of the oral cavity, any other coexisting malignancies, previous history of surgical treatment of the neck, radiotherapy or chemotherapy, and

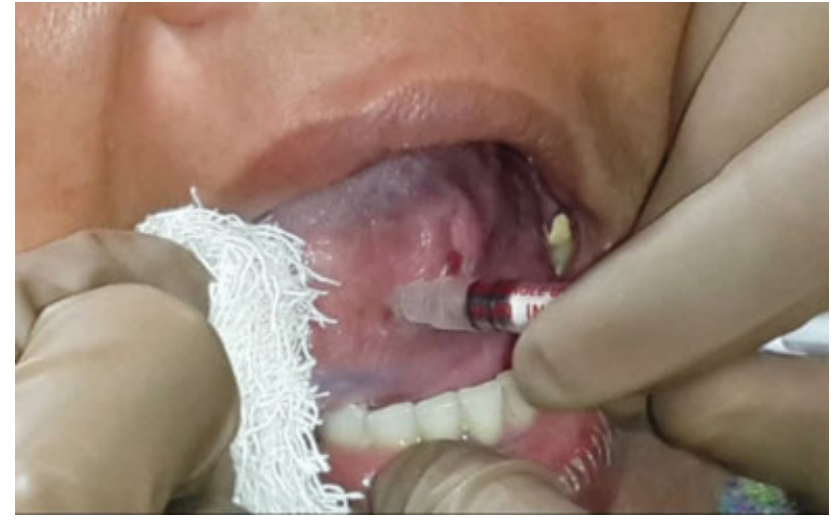

Fig. 1 Radiotracer being injected in the anterior quadrant of the primary tumor, which is located on the middle third of the left lateral border of the tongue.

patients unfit for surgery due to associated comorbidities were excluded from the study.

Technique: sentinel lymph node (SLN) biopsy-guided neck dissection + wide local excision (WLE) of the primary tumor.

A 4-quadrant peritumoral, submucosal injection of filtered Tc $99 \mathrm{~m}$ sulfur colloid radiotracer in a dose of 0.1 millicurie in each quadrant, and particle size of $220 \mathrm{~nm}$, was administered an hour prior to taking up the patient for surgery ( - Fig. 1). The radiotracer was procured from the Board of Radiation and Isotope Technology (BRIT), a unit of the Department of Atomic Energy of the government of India. Early dynamic and late static lymphoscintigraphy imaging followed by a SPECT-CT were performed, as shown in - Fig. 2. We used a Symbia T-16, (Siemens, Erlangen, Germany) dual-head gamma camera with a 16-slice CT scanner, for the lymphoscintigraphy and SPECT-CT. We also used a large Europrobe model CE0459 (EuroRad, Strasbourg, France) gamma probe. Once the patient underwent general anesthesia induction, the gamma probe was applied to the neck, and the neck levels with radioactivity were marked and documented as preflap counts, that is, before raising the subplatysmal flaps. Postflap in vivo counts, that is, after raising the subplatysmal flaps but before performing the neck dissection, were documented, as shown in - Fig. 3. The neck dissection of the patient was planned according to the sentinel node level obtained, and one level above and one level below the SLN were included in the neck dissection specimen. Exploration of levels IIB and $V$ was dependent upon the radioactivity in level IIA. Once the LN and the fibrofatty tissue from one level were dissected, the gamma probe was applied to the specimen, and postflap ex vivo counts were recorded, as shown in - Table 1, that is, the radioactivity of the $\mathrm{LN}$ after their resection. After completion of the neck dissection, the gamma probe was applied to the dissected neck, and the residual radioactivity was recorded as bed counts, after which the nodes were removed. In our study, radioactivity equal to at least double that of the background activity in the neck nodes was considered significant. All of the neck nodes and their levels were labeled before forwarding the specimen for histopathological examination. Sentinel nodes were not identified to the histopathologist to avoid bias.

Wide local excision of the primary tumor with $1-1.5 \mathrm{~cm}$ margin all around was performed, followed by primary 


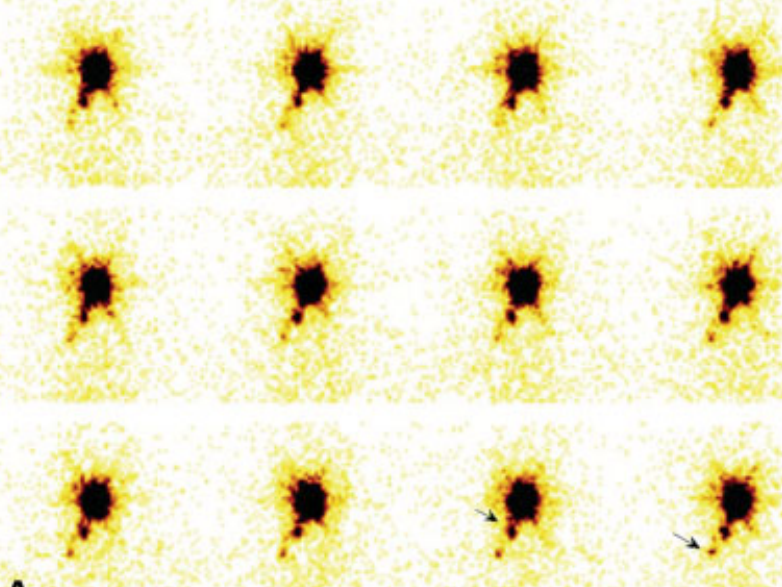

B

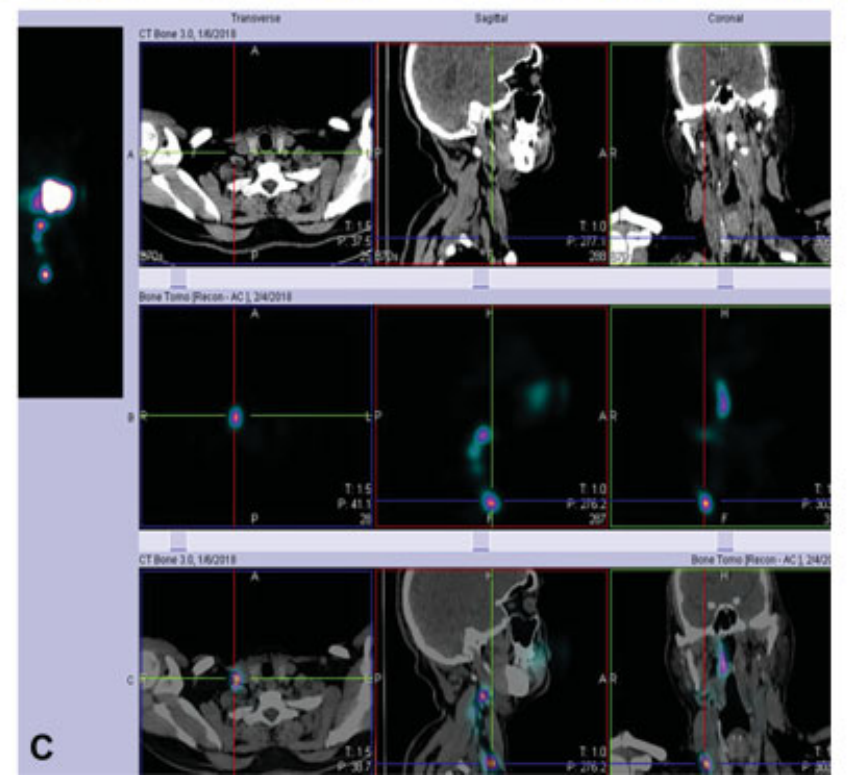

Fig. 2 Imaging in a case of Carcinoma right lateral border of the tongue with detection of first- and second-echelon nodes. (A) Early dynamic imaging (arrows showing the locaation of sentinel lymph nodes). (B) Late static imaging. (C) Single photon emission computed tomographycomputed tomography showing first-echelon node at level II and second-echelon node at level IV.
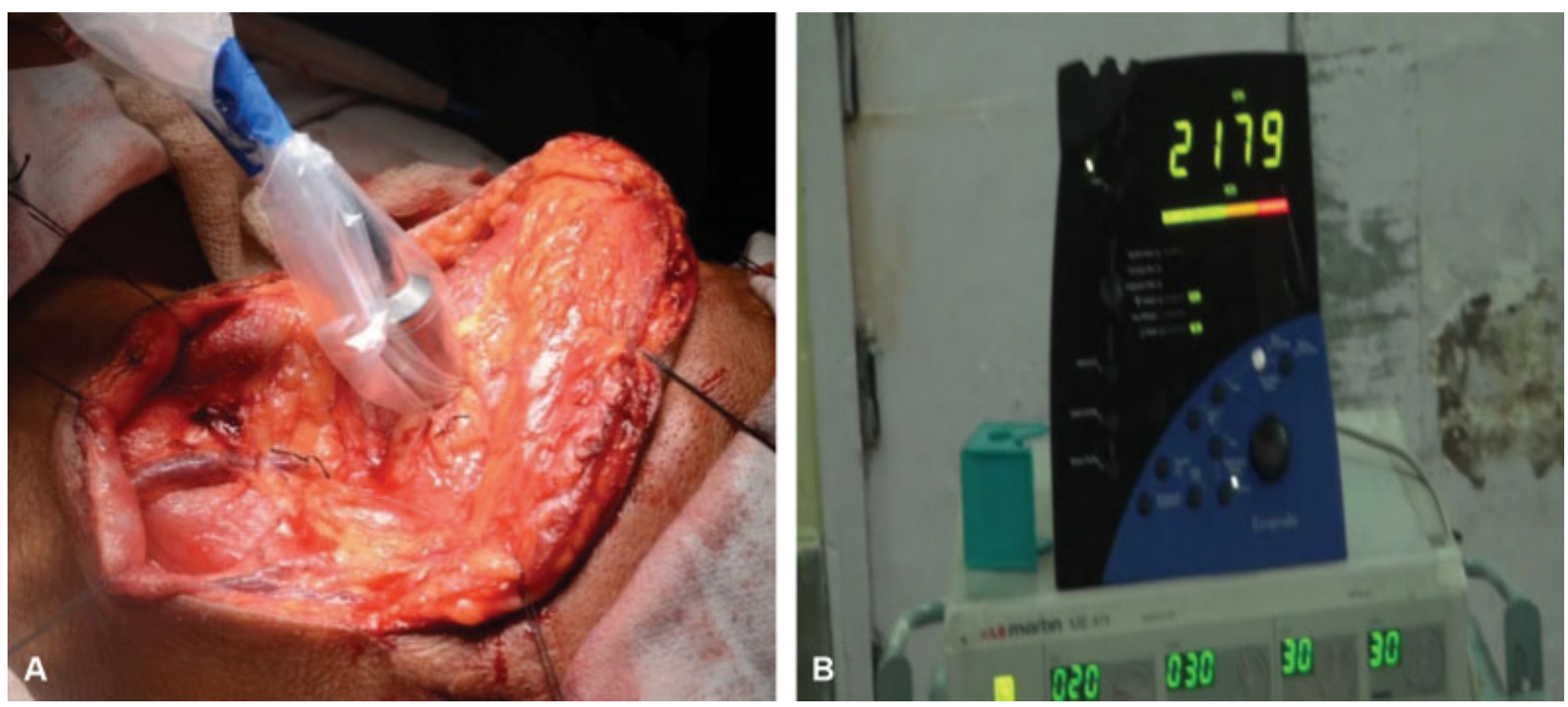

Fig. 3 (A) Gamma probe application after raising the subplatysmal flap. Postflap in vivo counts are recorded. (B). High radioactivity in the sentinel lymph node. 
Table 1 Documentation of radioactive tracer counts within the lymph nodes at various levels before and after raising the subplatysmal flap and before, during, and after the neck dissection

\begin{tabular}{|l|l|l|l|l|}
\hline $\begin{array}{l}\text { Neck } \\
\text { node } \\
\text { levels }\end{array}$ & Preflap & Postflap & \\
\hline & & In vivo & Ex vivo & Bed \\
\hline I A & $100 \mathrm{kc}$ & $1,200 \mathrm{kc}$ & Background & Background \\
\hline I B & $1,900 \mathrm{kc}$ & $\begin{array}{l}1,900 \mathrm{kc} \\
1,000 \mathrm{kc}\end{array}$ & $\begin{array}{l}2,000 \mathrm{kc} \\
500 \mathrm{kc}\end{array}$ & Background \\
\hline II A & $2,000 \mathrm{kc}$ & $1,500 \mathrm{kc}$ & $1,500 \mathrm{kc}$ & Background \\
\hline II B & - & Background & Background & Background \\
\hline III & Background & Background & Background & Background \\
\hline IV & 500 kc & $500 \mathrm{kc}$ & $500 \mathrm{kc}$ & Background \\
\hline V & Background & Background & Background & Background \\
\hline
\end{tabular}

Note: The highlighted node levels are the echelon levels detected with gamma probe. Abbreviation: kc, kilocounts.

closure or split-thickness skin graft cover, especially for the buccal mucosa primary. The histopathological examination of the LNs included grossing, hematoxylin and eosin staining, and serial-step sectioning with a minimum of 5 slices, each measuring up to 5 microns. In highly suspicious nodes with presence of micrometastases or sinus histiocytosis, immunohistochemistry (IHC) for pancytokeratin was performed. A revision histopathological examination with serial-step sectioning and IHC was performed on the sentinel nodes that were initially reported as negative for metastases.

\section{Statistical Analysis}

The analysis was conducted using the Statistical Package for the Social Sciences (SPSS) software, version 22.0 (IBM Corp., Armonk, NY, US). All point estimates were supplemented with their corresponding 95\% confidence intervals (95\%CIs). Continuous data, assumed to be normally distributed, was written in the form of their means and standard deviations (SD), and skewed data were written in the form of their medians and interquartile ranges, as per the requirement. Proportions were compared using the Chi-squared or Fisher exact test, depending on their applicability. All of the statistical tests were two-sided, and were performed at a significance level of $\alpha=0.05$. The diagnostic accuracy was measured by calculating the sensitivity, specificity, positive predictive value, and negative predictive value.

\section{Results}

The details of the demography, primary tumor and its characteristics, and type of neck dissection for both groups have been described in the - Table 2 .

The mean (SD) age of the study participants was 52.3 (16.3) years. Males outnumbered females with several 13 out of 20 (65\%). Addictions were present in 11 (55\%) patients, and the most common addiction was tobacco smoking. The most common oral cavity subsite was the lateral border of
Table 2 Demography and various parameters for the patients of the study and control groups

\begin{tabular}{|c|c|}
\hline Characteristics & Study group $(n=20)$ \\
\hline Age (mean $\pm S D$ in years) & $52.3 \pm 16.3$ \\
\hline \multicolumn{2}{|l|}{ Gender } \\
\hline Male & $13(65 \%)$ \\
\hline Female & 7 (35\%) \\
\hline \multicolumn{2}{|l|}{ Addictions } \\
\hline Present & $11(55 \%)$ \\
\hline Absent & $9(45 \%)$ \\
\hline \multicolumn{2}{|l|}{ Oral cavity subsite } \\
\hline Tongue & $13(65 \%)$ \\
\hline Buccal mucosa & $6(30 \%)$ \\
\hline RMT & $1(5 \%)$ \\
\hline \multicolumn{2}{|l|}{ TNM staging } \\
\hline \multicolumn{2}{|l|}{ Clinical } \\
\hline Stage I (T1N0M0) & $5(25 \%)$ \\
\hline Stage II (T2N0M0) & $15(75 \%)$ \\
\hline \multicolumn{2}{|l|}{ Pathological } \\
\hline $\mathrm{N}+$ & $6(30 \%)$ \\
\hline N1 & 4 \\
\hline $\mathrm{N} 2 \mathrm{~B}$ & 2 \\
\hline $\mathrm{N}-$ & $14(70 \%)$ \\
\hline \multicolumn{2}{|l|}{ Neck dissection } \\
\hline SND & 17 (85\%) \\
\hline MND & $3(15 \%)$ \\
\hline $\begin{array}{l}\text { Operating time } \\
\text { (mean } \pm \text { SD in hours) }\end{array}$ & $2.46 \pm 0.48$ \\
\hline Tumor depth & $\mathrm{n}=17$ \\
\hline Range in mm & $1-13$ \\
\hline Mean \pm SD in $\mathrm{mm}$ & $7.24 \pm 2.84$ \\
\hline Complication rate & $5 \%(1 / 20)$ \\
\hline Recurrence rate & NIL \\
\hline Death rate & $5 \%(1 / 20)$ \\
\hline
\end{tabular}

Abbreviations: MND, modified neck dissection; RMT, retromolar trigone; SD, standard deviation; SND, sentinel neck dissection; TNM, tumor, node, metastasis.

the tongue, which affected $65 \%$ of the sample, followed by the buccal mucosa, which affected $30 \%$, and the retromolar trigone (RMT), which affected $5 \%$ of the sample.

A total of 17 (85\%) patients underwent SND, and 3 (15\%) underwent modified neck dissection (MND). The mean (SD) operating time was of $2.46(0.48)$ hours. Tumor depth was not uniformly recorded for all patients in our study; therefore, the analysis of depth-related variables was excluded.

We have compared the SLN identification rate on lymphoscintigraphy, on SPECT-CT, and with gamma probe use. This is defined as the identification of at least one SLN in every patient. The SLN identification rate was the same with lymphoscintigraphy and SPECT-CT, that is, the echelon node 
Table 3 Showing the incidence of single and multiple sentinel lymph nodes, and the overall incidence of first and second echelons on imaging and gamma probe

\begin{tabular}{|l|l|l|}
\hline & Imaging $(\boldsymbol{n}=\mathbf{1 4 )}$ & $\begin{array}{l}\text { Gamma probe } \\
(\boldsymbol{n}=\mathbf{2 0})\end{array}$ \\
\hline Single SLN & $5(35.7 \%)$ & $8(40 \%)$ \\
\hline Multiple SLN & $9(64.2 \%)$ & $12(60 \%)$ \\
\hline Total & $14(99.9 \%)$ & $20(100 \%)$ \\
\hline First echelons & Imaging $(\boldsymbol{n}=14)$ & $\begin{array}{l}\text { Gamma probe } \\
(\boldsymbol{n}=20)\end{array}$ \\
\hline IB & $5(37.5 \%)$ & $11(55 \%)$ \\
\hline IIA & $8(57.14 \%)$ & $6(30 \%)$ \\
\hline III & $1(7.14 \%)$ & $3(15 \%)$ \\
\hline Total & $14(99.9 \%)$ & $20(100 \%)$ \\
\hline Second echelons & Imaging $(\boldsymbol{n}=9)$ & $\begin{array}{l}\text { Gamma probe } \\
(\boldsymbol{n}=12)\end{array}$ \\
\hline IIA & $3(33.3 \%)$ & $6(50 \%)$ \\
\hline IIB & - & $1(8.3 \%)$ \\
\hline III & $3(33.3 \%)$ & $3(25 \%)$ \\
\hline IV & $3(33.3 \%)$ & $2(16.6 \%)$ \\
\hline Total & $9(99.9 \%)$ & $12(99.9 \%)$ \\
\hline
\end{tabular}

Abbreviation: SLN, sentinel lymph node.

levels on lymphoscintigraphy correlated well with those on the SPECT-CT. Therefore, we have compared the identification rate on imaging with that of the gamma probe. The SLN identification rate with imaging alone was of $70 \%$, that is, 14 out of 20 patients had SLNs detected. The 6 patients whose SLNs were not located on the imaging exam had carcinoma of the buccal mucosa. The SLN identification rate with the the intraoperartive use of the gamma probe was of $100 \%$, that is, all 20 cases had $\geq 1$ SLN identified.

The time between the peritumoral injection of the radiotracer and the application of the gamma probe intraoperatively ranged from 90 minutes to 240 minutes, with a mean interval of $165.25 \pm 32.38$ minutes.

Upon imaging, out of 14 patients, a single sentinel node was detected in 5 (35.7\%), and multiple (that is, more than one) sentinel nodes were detected in 9 (64.2\%) patients. With the gamma probe, a single sentinel node was detected in 8 (40\%), and multiple nodes were detected in 12 (60\%) patients. We analyzed the overall incidence of first-, second-, and thirdechelon nodes at various levels in the present study, irrespective of the tumor subsite, as shown in - Table 3. The highest incidences of first-echelon node on imaging and gamma probe were at levels IIA and IB respectively. Second-echelon node was equally incident on imaging at levels IIA, III, and IV, whereas with gamma probe, level IIA was the most common. Thirdechelon nodes were detected only on gamma probe application in 5 tongue-cancer patients, with the highest incidence at level IV in 4 (80\%), followed by level III in 1 case (20\%).

We also analyzed the tumor subsite specific incidence of first- and second-echelon nodes with imaging and gamma probe, as shown in - Fig. 4. Out of 13 cases of carcinoma of the tongue, 4 (30.7\%) had first-echelon nodes detected at level IB, and 1 (7.7\%) had it at level III with both imaging and gamma probe, and the remaining $8(61.5 \%)$ patients had firstechelon nodes detected at level IIA on imaging. On gamma probe, of these 8 patients, 6 had first-echelon nodes detected at level IIA, and 2 patients had it detected at level III. Six cases of buccal mucosa had first-echelon nodes detected at level IB only on gamma probe application. One case of retromolar trigone had first-echelon node at level IB with both imaging and gamma probe.

The second-echelon level detection showed differences for tongue primary, with variable incidence on imaging and gamma probe. Nine (69.2\%) out of 13 cases of carcinoma of the tongue had second-echelon nodes detected. It was equally incident at levels IIA, III, and IV on imaging, whereas with gamma probe, it was most incident at level IIA, followed by levels III and IV and, in one case, at level IIB. Secondechelon node for buccal mucosa primary was detected in 3 patients only with gamma probe, and was most common at level IIA (66.6\%), followed by level III (33.3\%).

A total of 21 neck sides were examined in 20 patients ( 1 patient with tongue tip primary had bilateral neck dissection), and a total of 458 LNs were harvested, out of which 208 were the SLNs, with an average of 10.4 SLNs harvested per person. Out of the 458 LNs harvested, 25 (5.5\%) were metastatic, $20(80 \%)$ of which were SLNs, that is, the true positives. The remaining 5 (20\%) were non-SLNs, that is, the false negatives. Diagnostic accuracy was calculated in terms of their sensitivity, specificity, positive predictive value (PPV), and negative predictive value (NPV), which were of $80 \%, 56.58 \%, 9.61 \%$, and $98 \%$ respectively.

The complication rate was of $5 \%$ in our study. The most common complication noted was development of trismus, postoperatively. Death occurred in 1 (5\%) patient 4 months postoperatively due to age-related comorbidities. No locoregional recurrence was noted in the studied patients up to date.

\section{Discussion}

Observations pertaining to age and gender of the patients in this study were similar to the results of the study by De Carvalho et $\mathrm{al}^{7}$ which showed male preponderance and no statistical difference in terms of age distribution of the patients.

The most common oral cavity subsite involved by the primary tumor was the tongue, followed by the buccal mucosa. The subsite distribution of primary lesions was concurrent with the reviews of Warnakulasuriya et $\mathrm{al}^{8}{ }^{8}$ which state that the most common oral cavity subsite in oral cancer is the tongue in the European and US populations, whereas in the Asian population, it is the buccal mucosa.

The tumor, node, metastasis (TNM) staging was studied, and 15 out of 20 (75\%) were T2 NO M0, that is, stage II, and 5 (25\%) were T1 N0 M0, that is, stage I, preoperatively. Four out of 15 stage-II cases had nodal metastasis, with 3 upstaged to stage III and 1 upstaged to stage IVA. Two out of 5 stage-I cases had nodal metastasis, with 1 case each upstaged to stages III and IVA. There was upstaging to stages I and II by SLNB by $\sim 40 \%$ and $26.6 \%$ respectively. This was 

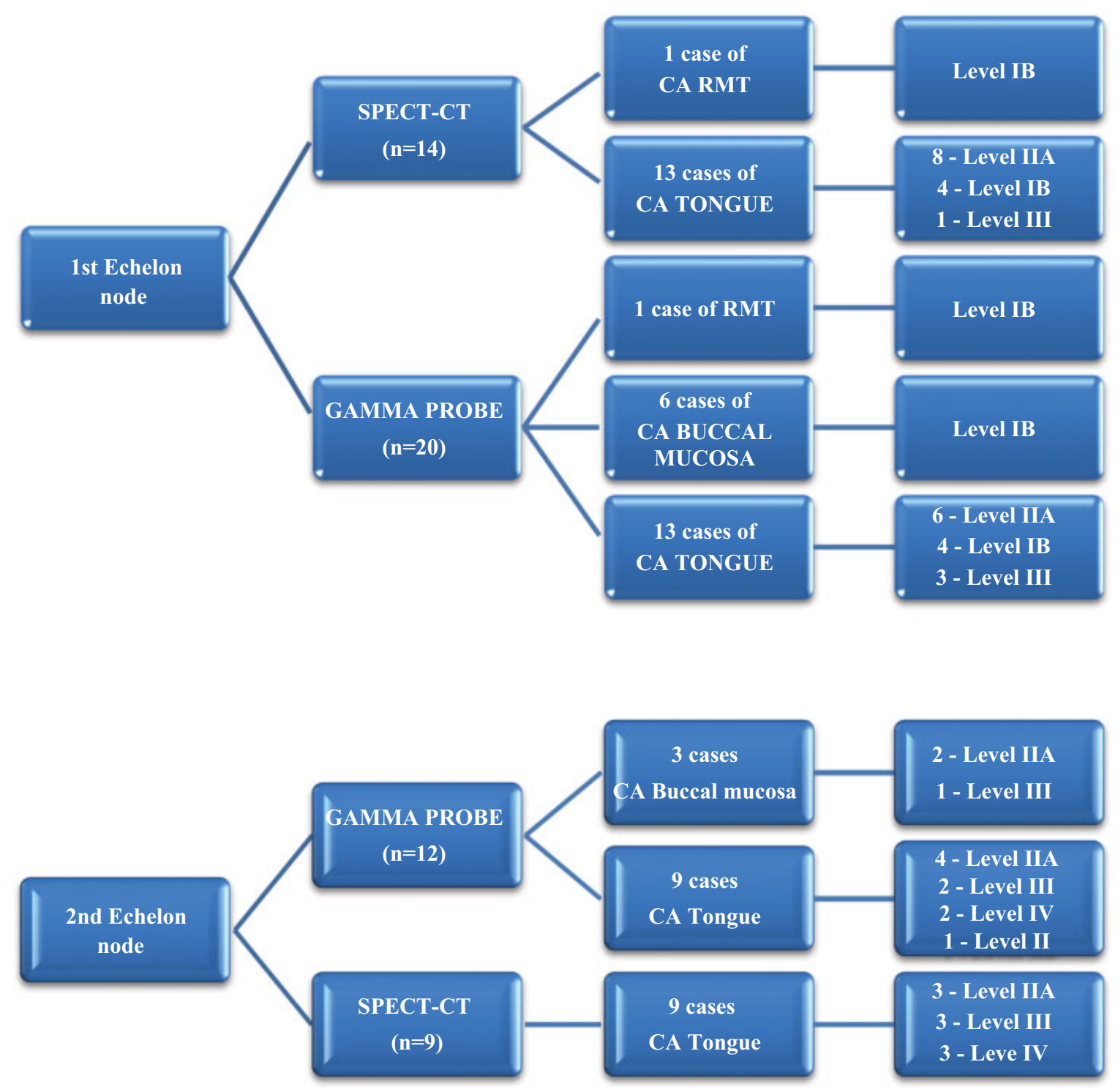

Fig. 4 (A) and (B) show the primary tumor subsite specific incidence of first- and second-echelon nodes at various levels on single photon emission computed tomography-computed tomography and gamma probe.

asynchronous with the results of the study by Alkureishi et $\mathrm{al}^{9}{ }^{9}$ in which they concluded that T-stage has significant correlation with nodal metastasis, and also showed a risk of upstaging by SLNB to T1 and T2 tumors of 30\% and 50\%, respectively.

The SLN identification rate in our study was of $100 \%$ with intraoperative gamma probe, and it was of $70 \%$ with imaging, which included lymphoscintigraphy and SPECT-CT, whereas in the study by Schilling et $\mathrm{al}^{10}{ }^{10}$ the SLN identification rate was of $99.5 \%$ with lymphoscintigraphy and gamma probe use. The SLN identification rate was the same when comparing lymphoscintigraphy and SPECT-CT, in contrast with the study by Toom et al, ${ }^{11}$ in which they concluded that use of SPECT-CT in addition to lymphoscintigraphy identified additional SLNs and upstaged the procedure. In our study, the $30 \%$ of patients whose SLNs were not detected on imaging were the 6 cases of buccal mucosa, whereas intraoperative gamma probe identified the SLNs in all of these cases. So far, no studies have been oerformed exclusively on SLNB of carcinoma of the buccal mucosa to determine its role. Moreover, the identification rate of multiple SLNs was better with intraoperative gamma probe use when compared with imaging.

We studied the incidence of first-, second-, and thirdechelon nodes in all of the patients undergoing SLNB, and compared them with the use of imaging and gamma probe. The first-, second-, and third-echelon nodes were determined by correlating the lymphoscintigraphy and SPECT-CT imaging with the intraoperative radioactive counts of the LNs upon application of the gamma probe.

The overall incidence of first-echelon node was the highest at level IIA with imaging, and at level IB with gamma probe use. While the subsite specific incidence showed the highest incidence at level IIA for tongue cancer on both imaging and probe use, and at level IB for buccal mucosa and RMT cases. In two cases of tongue carcinoma, there was a discrepancy in that the first echelon on imaging was level IIA, while on gamma probe it was level III. The reason for this 
could be the drainage of the radiotracer from its first-echelon node, which might have provided less radioactivity counts at level IIA and high counts at level III. This also applies to the difference in second-echelon levels in tongue cases in our study. This finding emphasizes the importance of having a shorter time interval between radiotracer injection and gamma probe application for best results, that is, the sooner the patient is taken up for surgery, the more precise would be the determination of various echelon nodes.

A special mention must be made of two cases of tongue primary in our study, in which one case had the first-echelon node at level III on both imaging and gamma probe, but histopathology showed no metastasis, and the other case had first-echelon node at level IIA and second-echelon node at level III, but histopathology showed metastasis in a level-III node, that is, skip metastasis.

The predictability of third-echelon nodes in our study only with gamma probe use but not on imaging emphasizes the time the radiotracer takes to drain through the lymphatics down the nodal basin. Therefore, early and late lymphoscintigraphy and SPECT-CT, which were performed within 30 minutes of radiotracer injection, could not pick up third-echelon nodes, whereas gamma probe applied intraoperatively did pick up the third-echelon nodes in 5 cases in which the time interval was prolonged, compared with other cases. So far, studies have shown the total number of SLNs detected, but none of have demonstrated in detail the various levels of echelon nodes in oral cancers.

Predetermination of neck node levels that were to be explored and dissected avoided the extensive exploration and dissection of the neck and decreased the operating time in the majority of the patients. Intraoperative gamma probe application differentiated the sentinel and non-sentinel LNs at a given echelon level based on the radioactivity that were all resected, levels labeled and sent for biopsy, and also it detected residual nodes in the dissected neck, which were also removed. The diagnostic accuracy of SLNB in our study in terms of SLN identification rate, sensitivity, specificity, positive predictive value (PPV), and negative predictive value (NPV) were pf $100 \%$, $80 \%, 56.58 \%, 9.61 \%$, and $98 \%$ respectively. The rates found in two of the biggest meta-analyses studying the diagnostic accuracy of SLNB in oral cancer were as follows: in the study by Hassan et al, ${ }^{12}$ the sensitivity, specificity, PPV, and NPV were of $93 \%, 100 \%, 100 \%, 97 \%$ respectively, and in the study by Liu et $a{ }^{13}$ the SLN identification rate, sensitivity, and NPV were of $96.3 \%, 87 \%$, and $94 \%$, respectively.

A revision histopathological examination by further serial slicing and IHC for pancytokeratin was performed in the SLNs reported negative for metastasis. It identified 2 patients with one positive SLN each; this increased the number of true positives and increased the sensitivity of the procedure from 78.2 to $80 \%$. The limitation of the present study is a small sample size, with a higher number of cases of carcinoma of the tongue and lower representation for other oral subsites, low specificity and PPV of the SLNB when compared with other studies.

\section{Conclusion}

High SLN identification rate, sensitivity, and NPV imply a definitive role of SLNB in the staging of node-negative necks in early-stage oral cancers. Radionuclide imaging with lymphoscintigraphy and SPECT-CT predicts the first-echelon neck levels, and, in some cases, second-echelon nodes as well, thus determining the neck levels to be explored. Intraoperative gamma probe application differentiates the sentinel and nonsentinel LNs within the echelon levels with the advantage of prediction of the next echelon levels if the time interval between radiotracer injection and surgery is long. The combined use of imaging and gamma probe certainly has high predictability for various echelon levels in early-stage oral cancers.

Conflicts of Interest

The authors have none to declare.

\section{References}

1 Woolgar JA. Detailed topography of cervical lymph-node metastases from oral squamous cell carcinoma. Int J Oral Maxillofac Surg 1997;26(01):3-9

2 Nieweg OE, Tanis PJ, Kroon BB. The definition of a sentinel node. Ann Surg Oncol 2001;8(06):538-541

3 Ziessman HA, O'Malley JP, Thrall JH. Oncology: Non-positron emission tomography. Nuclear medicine-the requisites. In: Fahey FH, editors. Philadelphia: Saunders Elsevier; 2014:281-283

4 Bluemel C, Herrmann K, Giammarile F, et al. EANM practice guidelines for lymphoscintigraphy and sentinel lymph node biopsy in melanoma. Eur J Nucl Med Mol Imaging 2015;42(11): 1750-1766

5 Ziessman HA, O'Malley JP, Thrall JH. Radiation detection and instrumentation. Nuclear medicine-the requisites. In: Fahey FH, editors. Philadelphia: Saunders Elsevier; 2014:42-44

6 Ziessman HA, O'Malley JP, Thrall JH. Radiopharmaceuticals. SinglePhoton Emission Computed Tomography, Positron Emission Tomography, and Hybrid Imaging. In: Fahey FH, editors. Philadelphia: Saunders Elsevier; 2014:56-57

7 De Carvalho GM, Dias VG, Kohler H, Chone CT, Guimaraes AC, Crespo AN. Sentinel Lymph Node Biopsy vs. Elective Neck Dissection in Patients with T1/T2 N0 Oral Squamous Cell Carcinoma: A Matched Pair Analysis. Int J Oral Craniofac Sci. 2016;2(01):047-051

8 Warnakulasuriya S. Global epidemiology of oral and oropharyngeal cancer. Oral Oncol 2009;45(4-5):309-316

9 Alkureishi LW, Ross GL, Shoaib T, et al. Does tumor depth affect nodal upstaging in squamous cell carcinoma of the head and neck? Laryngoscope 2008;118(04):629-634

10 Schilling C, Stoeckli SJ, Haerle SK, et al. Sentinel European Node Trial (SENT): 3-year results of sentinel node biopsy in oral cancer. Eur J Cancer 2015;51(18):2777-2784

11 den Toom IJ, van Schie A, van Weert S, et al. The added value of SPECT-CT for the identification of sentinel lymph nodes in early stage oral cancer. Eur J Nucl Med Mol Imaging 2017;44(06): 998-1004

12 Hassan O. Taha MS, Mehairy HE. Sentinel lymph node biopsy versus elective neck dissection in evaluation of cNO neck in patients with oral and oropharyngeal squamous cell carcinoma. Systematic review and meta-analysis study. Egypt J Ear Nose Throat Allied Sciences. 2015;16:25-34

13 Liu M, Wang SJ, Yang X, Peng H. Diagnostic Efficacy of Sentinel Lymph Node Biopsy in Early Oral Squamous Cell Carcinoma: A Meta-Analysis of 66 Studies. PLoS One 2017;12(01):e0170322 